\title{
Castración química en el perro mediante la aplicación intraepididimal de sustancias esclerosantes
}

\section{Chemical castration in dogs by the intra-epididymal application of sclerosing substances}

\author{
José Goicochea Vargas ${ }^{1,5}$, Yusepe Gomez Marín', Marcelo Ratto Fuster², \\ Alfonso Chavera Castillo ${ }^{3}$, Wilson Rondón Jorge ${ }^{1}$, Fidel Acosta Pachorro ${ }^{1}$, \\ Carlos Ballarte Zevallos ${ }^{4}$
}

\section{Resumen}

\begin{abstract}
Se evaluaron los efectos clínicos, parámetros espermáticos y lesiones macroscópicas e histológicas que ocasiona la castración química en perros mediante la aplicación de sustancias esclerosantes. Se establecieron tres grupos: $(\mathrm{G} 1, \mathrm{n}=10)$, gluconato de clorhexidina al 4\%, (G2, n=10), cloruro de benzalconio 4\% + cetrimida al $0.4 \%$, y (G3, control, $\mathrm{n}=5$ ) cloruro de sodio al $0.9 \%$, a dosis de $0.5-1.0 \mathrm{ml}$ por vía intraepididimaria (IEPD) y bilateral el día 0 . Los efectos se evaluaron los días $2,7,14,28$ y 56 pos-aplicación. La oligospermia manifiesta fue observada en 9/10 animales del G1 (90\%) y en 7/10 del G2 (70\%) al día 2 pos-aplicación, con un descenso crítico de la concentración espermática $\left(3.78 \pm 6.3 \times 10^{6} / \mathrm{ml}\right.$ y $0.95 \pm 0.89 \times 10^{6} / \mathrm{ml}$, respectivamente, motilidad de $0.10 \pm 0.32-0.20 \pm 0.42 \%$ con movimiento oscilatorio, sin avance progresivo y vitalidad de $0.30 \pm 0.67 \%-0 \%$ en ambos grupos. La azoospermia se detectó en todos los eyaculados de ambos grupos a partir del día 28, mientras que las variables espermáticas fueron normales en los perros del grupo control. Los cambios macro y microscópicos fueron hidrocele, hemorragia intra y extratubular, destrucción y necrosis de la pared tubular, siendo más severa en G2, atrofia de los túbulos seminíferos con reemplazo de tejido fibrótico, quistes y formación de espermiogranulomas a partir del día 7 en G2 y G1. La castración química se considera una alternativa para la esterilización masiva en perros.
\end{abstract}

Palabras clave: castración química, clorhexidina, benzalconio, cetrimida, perros

\footnotetext{
${ }^{1}$ Laboratorio de Cirugía y Biotecnología Reproductiva, Facultad de Medicina Veterinaria y Zootecnia, Universidad Nacional Hermilio Valdizán - UNHEVAL, Huánuco, Perú

${ }^{2}$ Instituto de Ciencia Animal, Universidad Austral de Chile, Valdivia, Chile

${ }^{3}$ Laboratorio de Histopatología Animal, Universidad Nacional Mayor de San Marcos, Lima, Perú

${ }^{4}$ Laboratorio Central, Universidad Nacional Hermilio Valdizán, Huánuco, Perú

${ }^{5}$ E-mail: goivar22@hotmail.com
}

Recibido: 5 de marzo de 2019

Aceptado para publicación: 25 de enero de 2020

Publicado: 11 de agosto de 2020 
The clinical effects, sperm parameters and macroscopic and histological lesions caused by chemical castration in dogs through the application of sclerosing substances were evaluated. Three groups were established: (G1, $n=10), 4 \%$ chlorhexidine gluconate, $(\mathrm{G} 2, \mathrm{n}=10), 4 \%$ benzalkonium chloride $+0.4 \%$ cetrimide, and $(\mathrm{G} 3$, control, $\mathrm{n}=5$ ) chloride of $0.9 \%$ sodium, at a dose of $0.5-1.0 \mathrm{ml}$ intra-epididymal (IEPD) and bilateral on day 0 . The effects were evaluated on days $2,7,14,28$ and 56 post-application. The manifest oligospermia was observed in 9/10 animals of G1 (90\%) and in 7/10 of G2 (70\%) at day 2 post-application, with a critical decrease of sperm concentration $\left(3.78 \pm 6.3 \times 10^{6} / \mathrm{ml}\right.$ and $0.95 \pm 0.89 \times 10^{6} / \mathrm{ml}$, respectively, motility of $0.10 \pm 0.32-0.20 \pm 0.42 \%$ with oscillatory movement, without progressive advance and vitality of $0.30 \pm 0.67 \%-0 \%$ in both groups. Azoospermia was detected in all ejaculates of both groups from day 28, while sperm variables were normal in the dogs of the control group. The macro and microscopic changes were hydrocele, intra and extratubular haemorrhage, destruction and necrosis of the tubular wall, being more severe in G2, atrophy of the seminiferous tubules with replacement of fibrotic tissue, cysts and formation of spermiogranulomas from day 7 on G2 and G1. Chemical castration is considered an alternative for mass sterilization in dogs.

Key words: chemical castration, clorexidine, benzalkoniumm cetrimide, dogs

\section{INTRODUCCIÓN}

Diversos métodos han sido empleados para el control de la natalidad en los caninos. En el caso de los machos, la castración química es un método que consisten en la administración de sustancias esclerosantes por vía intra-testicular o intra-epididimal (Pineda y Hepler, 1978; Goicochea et al., 2004; Soto et al., 2009) o intraepididimal (Pineda et al., 1977; Pineda y Hepler, 1978; Goicochea et al., 2004; Leoci et al., 2019), con el objetivo de provocar lesiones permanentes del parénquima testicular y la obliteración del epidídimo con la consecuente azoospermia, evitando así las desventajas de la cirugía, como costo y cuidados postoperatorios (Freeman y Coffey, 1993; Soto et al., 2009).

El objetivo de este trabajo fue detallar las lesiones a nivel del epidídimo, los cambios en los parámetros espermáticos (volumen, concentración, motilidad y vitalidad espermática), alteraciones del volumen testicular y epididimario, lesiones macroscópicas y mi- croscópicas y el grado de dolor que ocasionan la clorhexidina $4 \%$ y el cloruro de benzalconio $4 \%$ + cetrimida al $0.4 \%$ cuando son aplicadas vía intraepididimal por aproximación percutánea.

\section{Materiales y Métodos}

Se seleccionaron al azar 25 perros vagabundos, con testículos normales, clínicamente sanos, de edades entre 2.5 y 7 años $(4.5 \pm 1.0)$ y pesos entre 6 y $25 \mathrm{~kg}(13.64 \pm$ $5.65)$, y de diverso tipo racial. Los perros fueron distribuidos aleatoriamente en tres grupos experimentales: Grupo 1: solución de gluconato de clorhexidina 4\% ( $\mathrm{n}=10)$; Grupo 2: cloruro de benzalconio $4 \%+$ cetrimida al $0.4 \%(\mathrm{n}=10)$; Grupo 3 (Control): suero fisiológico al 9\% $(n=5)$. Los animales fueron tranquilizados con $1 \mathrm{mg} / \mathrm{kg} / \mathrm{IM}$ de xilacina al $2 \%$. Luego se aplicó 0.5 a $1.0 \mathrm{ml}$ vía intraepididimal (IEPD) bilateral de la sustancia química esclerosante, empleando jeringa de tuberculina y aguja $26 \mathrm{Gx} 5 / 8$ ". Los animales 
Cuadro 1. Variables espermáticas (promedio \pm d.e.) de eyaculados de perros químicamente vasectomizados mediante la aplicación intraepididimal de gluconato de clorhexidina al 4\% (G1), cloruro de benzalconio al $4 \%+$ cetrimida al $0.4 \%(\mathrm{G} 2)$, y con cloruro de sodio $0.9 \%$ (G3, grupo control)

\begin{tabular}{lccc}
\hline & G1 & G2 & G3 \\
\hline Eyaculados $(\mathrm{n})$ & 37 & 37 & 20 \\
Volumen $(\mathrm{ml})$ & $2.6 \pm 1.3^{\mathrm{a}}$ & $1.6 \pm 0.8 \mathrm{~b}$ & $1.6 \pm 0.8^{\mathrm{b}}$ \\
Concentración & $107.0 \pm 192.3^{\mathrm{b}}$ & $68.9 \pm 176.6^{\mathrm{b}}$ & $294.48 \pm 169.5^{\mathrm{a}}$ \\
espermática $\left(10^{6} / \mathrm{ml}\right)$ & $24.1 \pm 40.1^{\mathrm{a}}$ & $23.0 \pm 38.3^{\mathrm{a}}$ & $85.2 \pm 6.3^{\mathrm{b}}$ \\
Motilidad & $24.8 \pm 41.3^{\mathrm{a}}$ & $24.1 \pm 40.2^{\mathrm{a}}$ & $94.0 \pm 07^{\mathrm{b}}$ \\
\hline Vitalidad &
\end{tabular}

${ }^{a, b}$ Letras diferentes dentro de una misma fila indican diferencias significativas $(p<0.05)$

fueron evaluados en el día 0 (día del tratamiento) y a $\operatorname{los} 2,7,14,28$ y 56 días posaplicación.

Los eyaculados (primera y segunda fracciones) se recolectaron a través de la manipulación digital y sin presencia de perra celadora. Los parámetros seminales fueron determinados mediante el espermiograma convencional. El volumen testicular se calculó mediante la fórmula empírica propuesta por Lambert (1951): $\mathrm{VT}=$ longitud (L) $\mathrm{x}$ ancho (A) x altura $(\mathrm{H}) \times 0.71$, y el volumen del epidídimo empleando la fórmula para un probable esferoide: $\mathrm{VEp}=4.1888 \times(\mathrm{L} / 2) 2 \times(\mathrm{H} /$ 2 ) $/ 1000$, donde las medidas fueron determinadas mediante ultrasonografía empleando un ecógrafo Aloka Prosound 6, con transductor lineal de $13 \mathrm{Mhz}$

La vitalidad se determinó a través de la tinción supravital con eosina-nigrosina (tinción de Blom) y la algesia se evaluó durante los primeros siete días pos-aplicación mediante la escala modificada de Waterman-Pearson (1999), el cual evalúa seis parámetros (pos- tura, actividad locomotriz, vocalización, atención al testículo, comportamiento y respuesta a la manipulación testicular) asignándole un puntaje de $0-3$ para cada parámetro, en una escala que va de 0 a 18, donde 0 : ausencia de dolor, 1 - 6: leve, 7 - 12: moderado y 13 - 18: severo.

Para el estudio histológico se seleccionó aleatoriamente un animal por tratamiento por día de evaluación y se realizó una orquiectomía pre-escrotal. Las muestras del epidídimo y testículo fueron procesadas según Luna (1968).

Los resultados fueron analizados mediante análisis de varianza, utilizando los modelos lineales generales (PROCGLM) del paquete estadístico SAS/STAT (1999) y las pruebas de comparación múltiple (HSD de Tukey) con $\mathrm{p}<0.05$ de confianza. El volumen, motilidad y vitalidad espermática como variables dependientes expresados en porcentaje fueron transformados al arcoseno para los análisis estadísticos respectivos. 

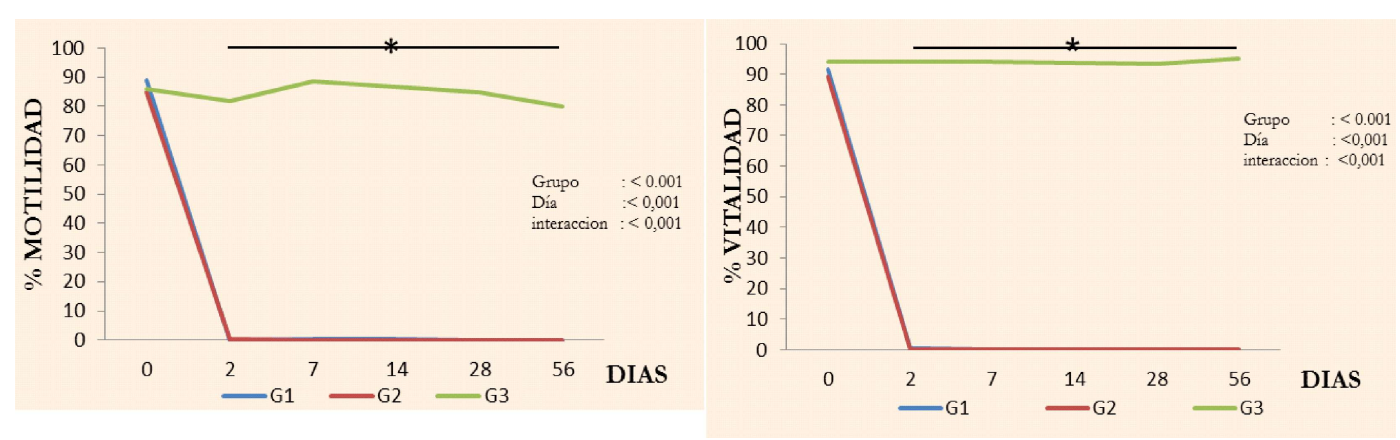

Figura 1. Motilidad (A) y vitalidad espermática (B) en perros sometidos a vasectomía química con gluconato de clorhexidina al $4 \%(\mathrm{G} 1)$, cloruro de benzalconio al $4 \%+$ cetrimida al $0.4 \%$ (G2) y cloruro de sodio al $0.9 \%$ - Grupo control (G3). p $<0.001$

Cuadro 2. Volumen testicular (promedio \pm DE) de perros químicamente vasectomizados mediante la aplicación intraepididimal de gluconato de clorhexidina al 4\% (G1), cloruro de benzalconio al 4\% + cetrimida al $0.4 \%$ (G2), y cloruro de sodio $0.9 \%$ (G3, Control)

\begin{tabular}{lccc}
\hline & G1 & G2 & G3 \\
\hline Volumen testicular $(\mathrm{ml})$ & $11.1 \pm 4.0^{\mathrm{a}}$ & $8.4 \pm 3.4^{\mathrm{b}}$ & $8.6 \pm 2.4^{\mathrm{b}}$ \\
Volumen epididimario $(\mathrm{ml})$ & $1.0 \pm 0.6^{\mathrm{a}}$ & $0.9 \pm 0.6^{\mathrm{a}}$ & $1.1 \pm 1.3^{\mathrm{a}}$ \\
\hline
\end{tabular}

${ }^{a, b}$ Letras diferentes dentro de una misma fila indican diferencias significativas $(p<0.05)$

\section{Resultados y Discusión}

\section{Volumen y Concentración Espermática}

Se hizo el análisis espermático de 94 eyaculados. Se observó un efecto de tratamiento $(\mathrm{p}=0.001)$ y tiempo $(\mathrm{p}=0.003)$ con relación a la variación del volumen del eyaculado y concentración espermática, pero no existió interacción entre estas dos variables $(\mathrm{p}=0.9)$ (Cuadro 1).

El volumen del eyaculado disminuyó significativamente $(\mathrm{p}<0.05)$ en los perros de los grupos G2 y G3 (Cuadro 1). De igual ma- nera, la concentración espermática fue significativamente menor en comparación con el grupo testigo $(p<0.05)$, pero similar entre ellos. El descenso de la concentración espermática ocurrió de manera abrupta a los 2 días de la aplicación, con una reducción del $98 \%$ (G1) y $99 \%$ (G2) afectando a $9 / 10$ y 7/10 de animales, respectivamente, y sin comprometer la libido ni la erección peneana. La concentración espermática fue menor que cuando se aplicó gluconato de zinc en dimetil sulfóxido (DMSO) al $0.5 \%$ por vía intratesticular (Soto et al., 2009), lo cual podría impedir que estos perros pudieran dejar gestantes a las hembras, a diferencia de lo que ocurre cuando se aplican otros compues- 

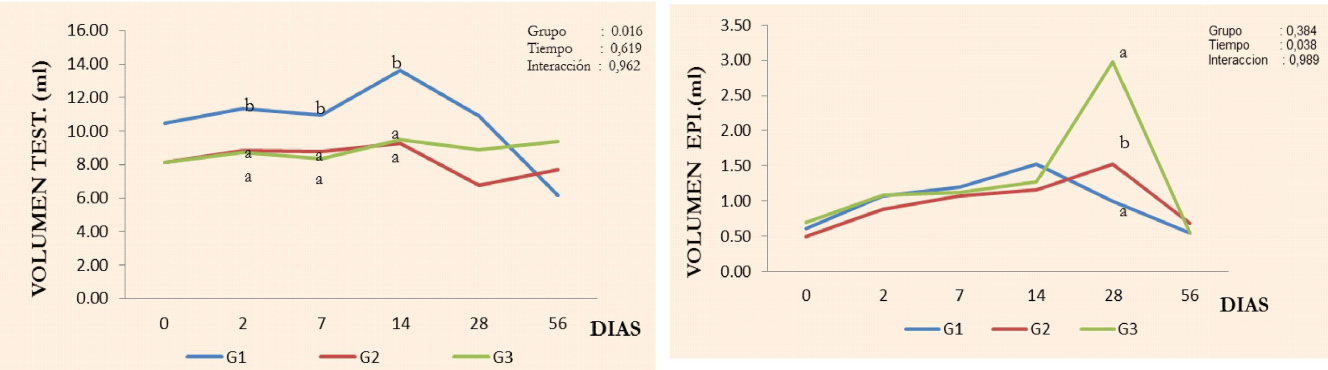

Figura 2. Volumen testicular (A) y epididimario (B) durante el periodo experimental en en perros sometidos a vasectomía química con gluconato de clorhexidina al $4 \%$ (G1), cloruro de benzalconio al $4 \%$ + cetrimida al $0.4 \%$ (G2) y cloruro de sodio al $0.9 \%$ Grupo control (G3). $\mathrm{p}<0.001$

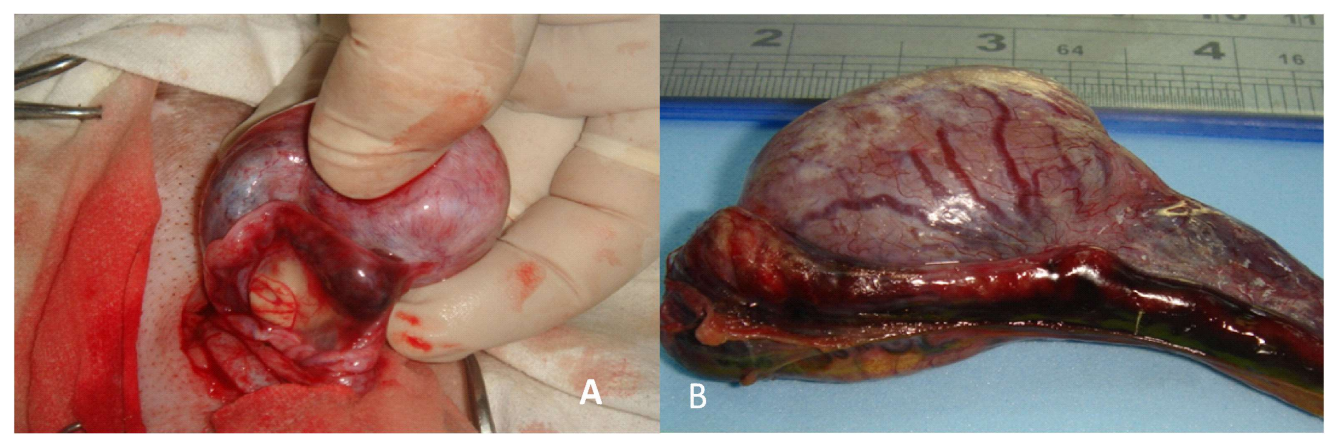

Figura 3. Área hemorrágica de la cola del epidídimo, día 2 pos-aplicación intra-epididimaria de gluconato de clorhexidina $4 \%$ (A) y cloruro de benzalconio al $4 \%+$ cetrimida al $0.4 \%$ (B)

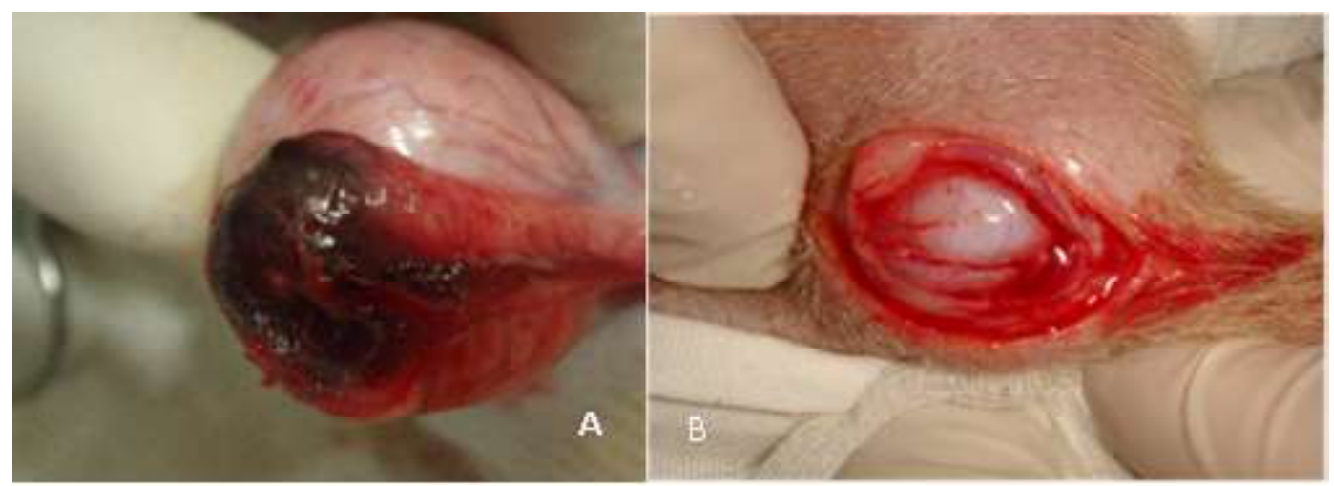

Figura 4. Lesión hemorrágica de la cola del epidídimo, día 7 pos-aplicación intra-epididimaria de gluconato de clorhexidina $4 \%$ (A). Hidrocele, producido por el cloruro de benzalconio al $4 \%+$ cetrimida al $0.4 \%$ (B) 

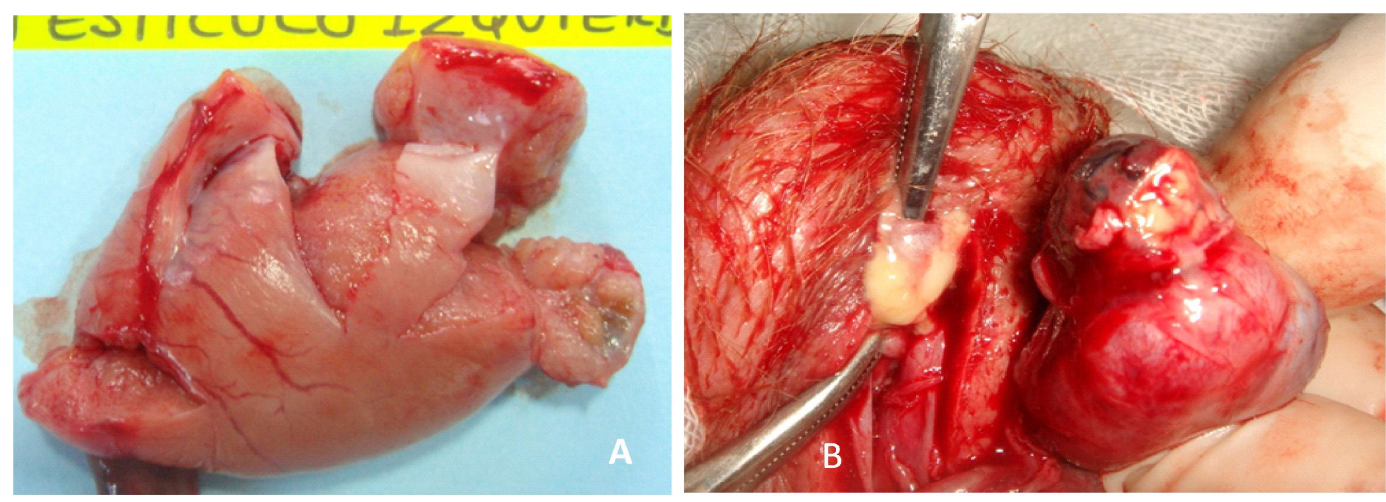

Figura 5. Presencia de tejido esclerosado en la cola del epidídimo producida por el gluconato de clorhexidina 4\% 28 días pos-aplicación (A). Exudado purulento separado de la cola del epidídimo producida por el cloruro de benzalconio al $4 \%+$ cetrimida al $0.4 \%$ (B)
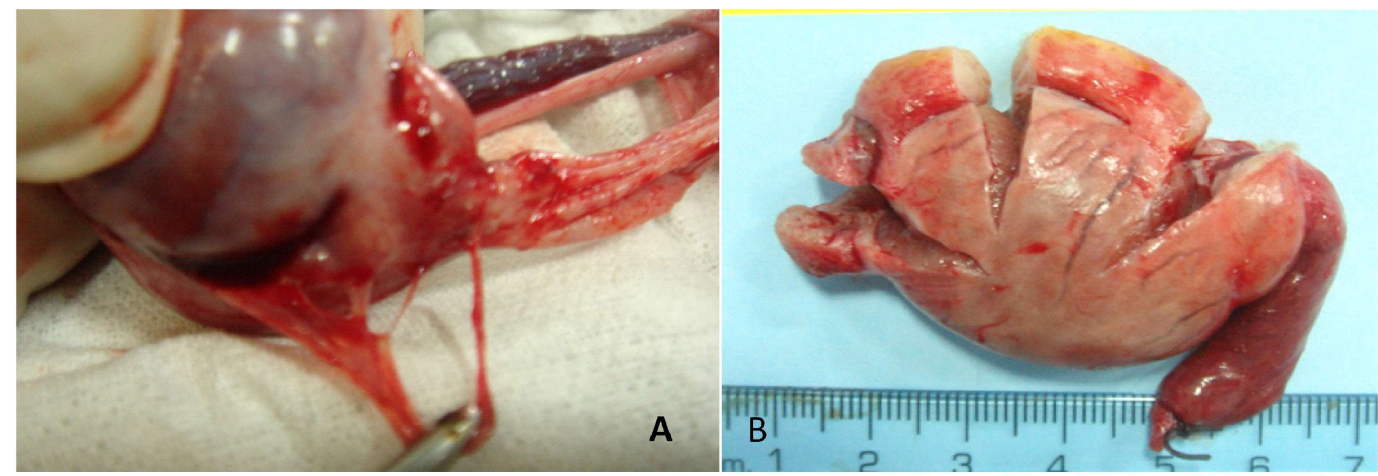

Figura 6. Adherencias de la cola del epidídimo a la túnica 56 días después de la aplicación de gluconato de clorhexidina 4\% (A). Esclerosis producida por el cloruro de benzalconio al $4 \%$ + cetrimida al $0.4 \%$ (B)

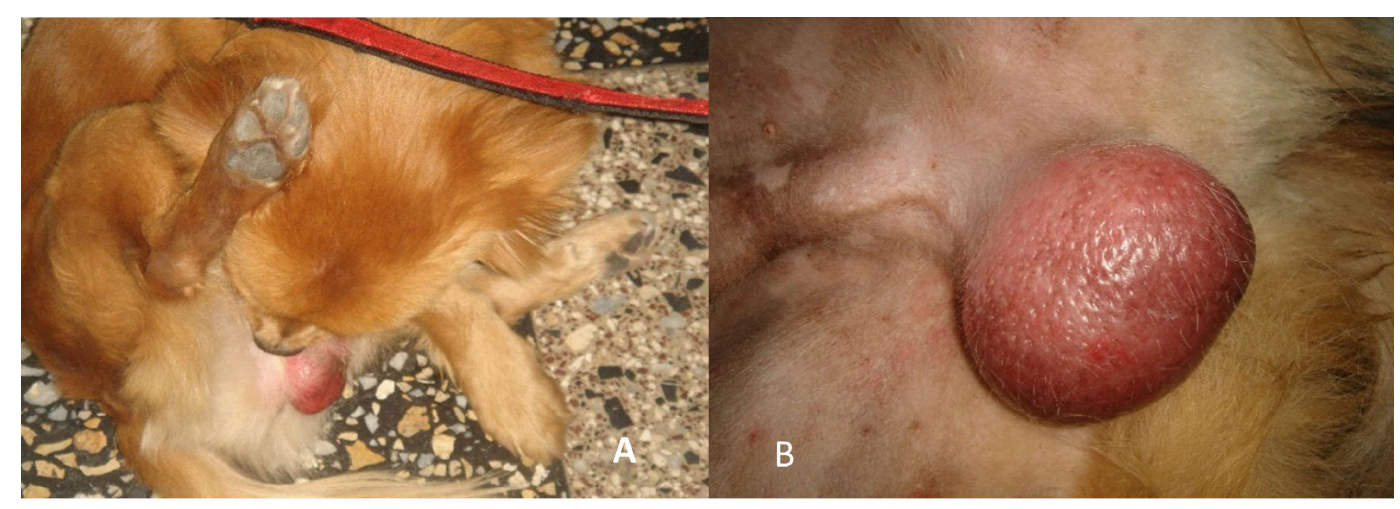

Figura 7. Lamido de escroto (A) y edema escrotal (B) detectado en algunos animales al $5^{\circ}$ día después de la aplicación de cloruro de benzalconio al $4 \%+$ cetrimida al $0.4 \%$ 
tos químicos (Koger, 1978), que además alteran el comportamiento sexual debido a una disminución de los niveles de testosterona (Tepsumethanon et al., 2005).

La azoospermia franca detectada a partir del día 28 fue producida por la intensa fibrosis con formaciones quísticas y espermiogranuloma en la cola epididimal (Figura 1) y que ocurre más tempranamente que cuando se aplica clorexidina en $50 \%$ de DMSO (Pineda et al., 1977).

\section{Motilidad y Vitalidad Espermática}

Se observó un efecto de tratamiento $(\mathrm{p}=0.001)$, tiempo $(\mathrm{p}=0.001)$ e interacción $(\mathrm{p}=0.001)$ con relación a la motilidad y vitalidad espermática, donde los perros de los grupos 1 y 2 mostraron una disminución drástica de estas variables desde el día 2 hasta el día 56 del tratamiento (Cuadro 1; Figura 1).

El porcentaje de motilidad espermática previo al tratamiento de los tres grupos experimentales estuvo dentro de los rangos normales, disminuyendo de manera manifiesta entre el día 7 y 14 en todos los animales de los grupos G1 y G2, a excepción de un perro de G1, quien presentó escasos espermatozoides vivos y pobre motilidad, siendo considerado infértil de acuerdo con Oettle (1993).

Si bien se detectó una escasa presencia de espermatozoides en los eyaculados desde el día 2 hasta el día 28 de la aplicación en los grupos G1 y G2, la mortalidad fue del 100\%, a excepción de dos de perros de G1 quienes presentaron una vitalidad de $1-2 \%$.

\section{Volumen Testicular y Epididimario}

El volumen testicular disminuyó significativamente $(p<0.05)$ en los perros tratados del G2 y G3, mientras que el volumen epididimario no fue afectado por el tratamiento (Cuadro 2; Figura 2). Se apreció un leve incremento del volumen testicular al $2^{\circ}$ día pos-aplicación que se extendió hasta el día
14, para luego disminuir significativamente $(p<0.05)$ en ambos grupos, en comparación con la orquiectomía química que provocan una mayor área inflamatoria y mayor agresión al parénquima testicular (Immegart y Threlfall, 2000). Asimismo, el volumen epididimario incrementó debido al proceso inflamatorio extensivo y difuso inicial (Figura 2), exacerbada por el incremento de la presión intraluminal producida por la deposición de las sustancias inyectadas; no obstante, este incremento no fue diferente del observado en el grupo control.

\section{Lesiones Macroscópicas}

El edema escrotal y hemorragia de la cola del epidídimo (Figuras 3 y 4 ) e hidrocele fueron los hallazgos más importantes detectados al día 2 del experimento, siendo más evidentes en los animales del G1. El hidrocele se mantuvo en casi todos los animales hasta el día 28, mientras que la hemorragia estuvo presente en algunos animales hasta el día 14. Además, la presencia de fibrosis de la túnica vaginal y albugínea (Figura 5) y de adherencias (Figura 6), fueron detectadas a partir del día 7 hasta el día 56, y la esclerosis del epidídimo fue apreciada entre el día 14 al 56 postratamiento (Figura 5). Por otro lado, el día 28 se apreció, en algunos animales de ambos grupos, un proceso inflamatorio purulento de la cola del epidídimo (Figura 5B).

Los signos clínicos inflamatorios producidos por la aplicación del gluconato de clorexidina al $4 \%$ (G1) y el cloruro de benzalconio al $4 \%$ + cetrimida $0.4 \%$ (G2) no solo comprometieron la integridad de la cola del epidídimo, sino también al testículo y las estructuras adyacentes, siendo el hidrocele, con presencia moderada de fluido seroso, el hallazgo más frecuente. Asimismo, la hemorragia fue un signo también manifiesto en algunos animales de ambos grupos (G1, G2), la cual fue observada hasta el día 14 acompañada de adherencias entre los días 7 y 56. Las lesiones encontradas en este estudio fueron similares a las comunicadas en perros so- 

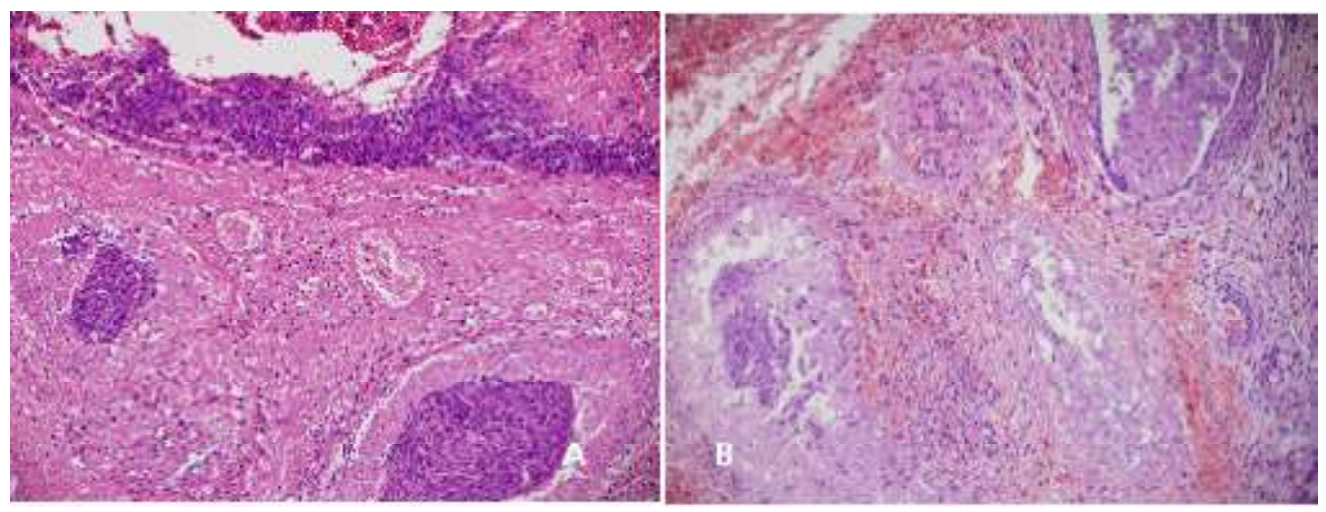

Figura 8. Cola del epidídimo 2 días después del tratamiento. A: Destrucción total de la pared tubular (200x), aplicación de gluconato de clorhexidina 4\%; B: Necrosis, hemorragia severa e infiltración de células inflamatorias (200x), cloruro de benzalconio al 4\% + cetrimida al $0.4 \%$. Tinción Hematoxilina-eosina

metidos a orquiectomía química (Goicochea et al., 2004; Levy et al., 2008; Soto et al., 2009).

\section{Lesiones Microscópicas}

Las lesiones microscópicas estuvieron circunscritas a la cola del epidídimo, permaneciendo el cuerpo y la cabeza sin lesiones. En el día 2 (Figura 8), se observó severa hemorragia con presencia de células inflamatorias (polimorfonucleares) en G1, como hallazgo más importante. Asimismo, se apreció destrucción de la pared de algunos túbulos epididimarios, otros con pared delgada y necrosada, túnica albugínea delgada y la externa engrosada y hemorrágica. Así mismo, en muestras de G2 (Figura 8B) se encontró inflamación y necrosis severa, submucosa tubular, media y externa sumamente engrosada, con mucosa epitelial descamada y obstruida.

Estas lesiones son producidas por el efecto altamente irritante de las sustancias químicas evaluadas (Hurber et al., 1988; Thomson; 2002). Por otro lado, el incremento de la presión en la cola del epidídimo ejercida durante la administración de las sustancias químicas podría haber contribuido al pro- ceso hemorrágico/inflamatorio inicial. No obstante, la necrosis de la pared de los túbulos y el engrosamiento y hemorragia de la túnica externa observada en G2 indica que el cloruro benzalconio al $4 \%$ + cetrimida $0.4 \%$ induce a una mayor necrosis y descamación del epitelio tubular (Figura 8B).

En los perros de G1 en el día 7 (Figura 9A) se aprecian rezagos de hemorragia, así como una extensión del proceso inflamatorio, epitelio destruido, túbulos epididimarios dilatados, túnica albugínea fibrosada, descamación y necrosis. Asimismo, en G2 (Figura 9B) se observa una reacción inflamatoria severa de tipo supurativo, pérdida de la arquitectura tubular, destrucción total de los túbulos, mucosa, submucosa y media y pared epitelial delgada con presencia de células vacuoladas.

Hacia el día 14 (Figura 10A), el proceso inflamatorio de moderado a severo continúa en G1. Se observa necrosis supurativa hemorrágica, incremento de tejido fibrótico peritubular, túbulos dilatados y formaciones quísticas con presencia de espermiogranulomas. En G2 (Figura 10B) se observa una reacción inflamatoria severa, supurativa di- 

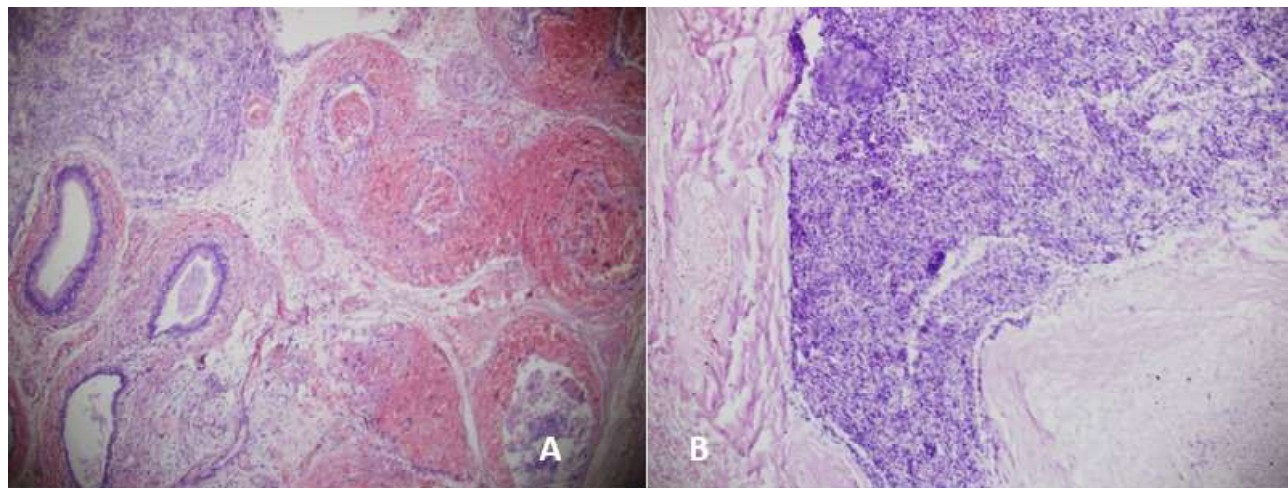

Figura 9. Cola del epidídimo 7 días después del tratamiento. A: Necrosis y hemorragia intra y extratubular (100x), gluconato de clorhexidina 4\%; B: Espermiogranuloma (200x), cloruro de benzalconio al $4 \%$ + cetrimida $0.4 \%$. Tinción Hematoxilina-eosina
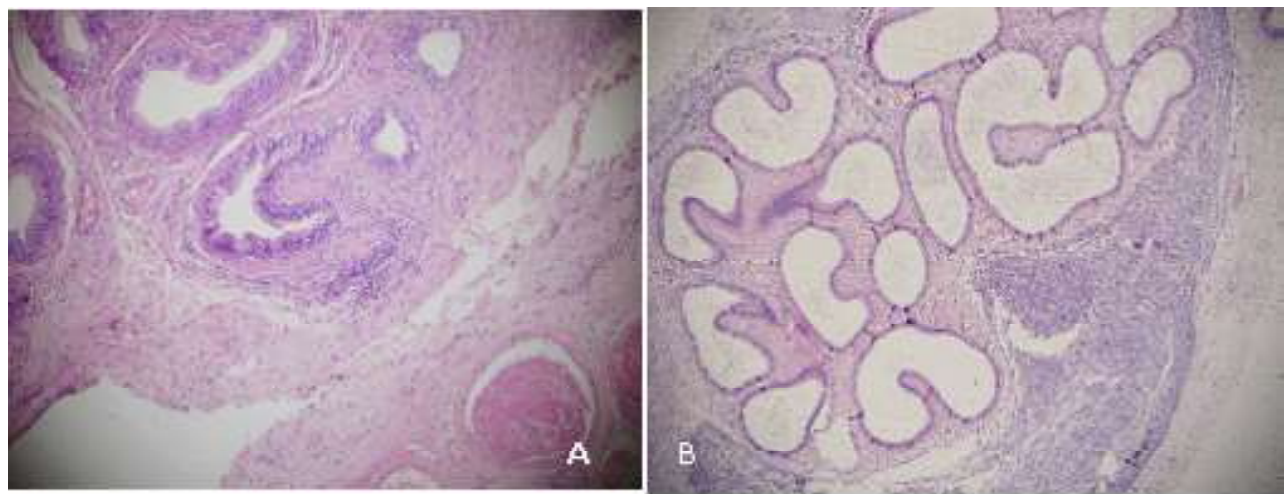

Figura 10. Cola del epidídimo, 14 días después del tratamiento. A: Túbulos con pared engrosada y fibrosis externa (100x), gluconato de clorhexidina $4 \%$. B: Túbulos dilatados, fibrosis y espermiogranuloma (40x), cloruro de benzalconio al $4 \%+$ cetrimida $0.4 \%$. Tinción Hematoxilina-eosina

fusa extensiva, túbulos epididimales irregulares con pared delgada, presentando extensa y severa fibrosis, con numerosos y grandes espermiogranulomas. Además, adherencias fibrosas con la túnica albugínea testicular.

El día 28 (Figura 11A), los animales del G1 presentan quistes tubulares con material inflamatorio y espermático, espermiogra- nulomas, con pared alineada por células aplanadas y la externa gruesa y fibrosa. Los animales del G2 (Figura 9A) presentan la cola del epidídimo severamente dañada, con hemorragia y necrosis, túnicas engrosadas y la externa fibrosada y hemorrágica, células inflamatorias con presencia de células mononucleadas y fibrina, presencia de áreas de espermiogranulomas. 

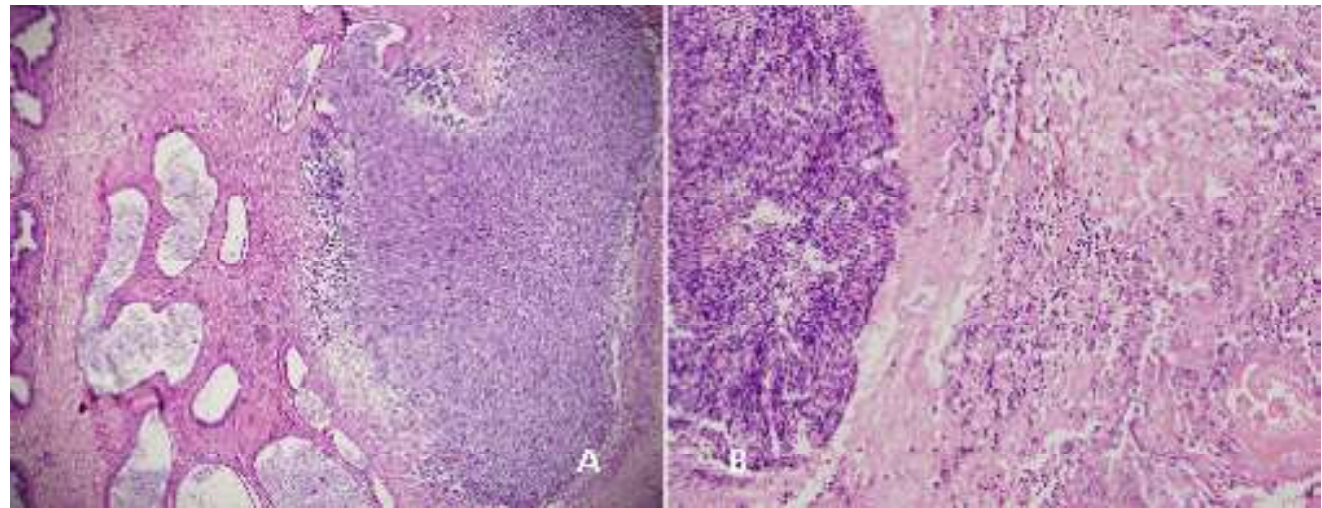

Figura 11: Cola del epidídimo, 28 días después del tratamiento. A: túbulos quísticos, fibrosis y gran espermiogranuloma (40x), gluconato de clorhexidina 4\%; B: Infiltración de polimorfonucleares y espermiogranuloma, cloruro de benzalconio al $4 \%+$ cetrimida $0.4 \%$. Tinción Hematoxilina-eosina

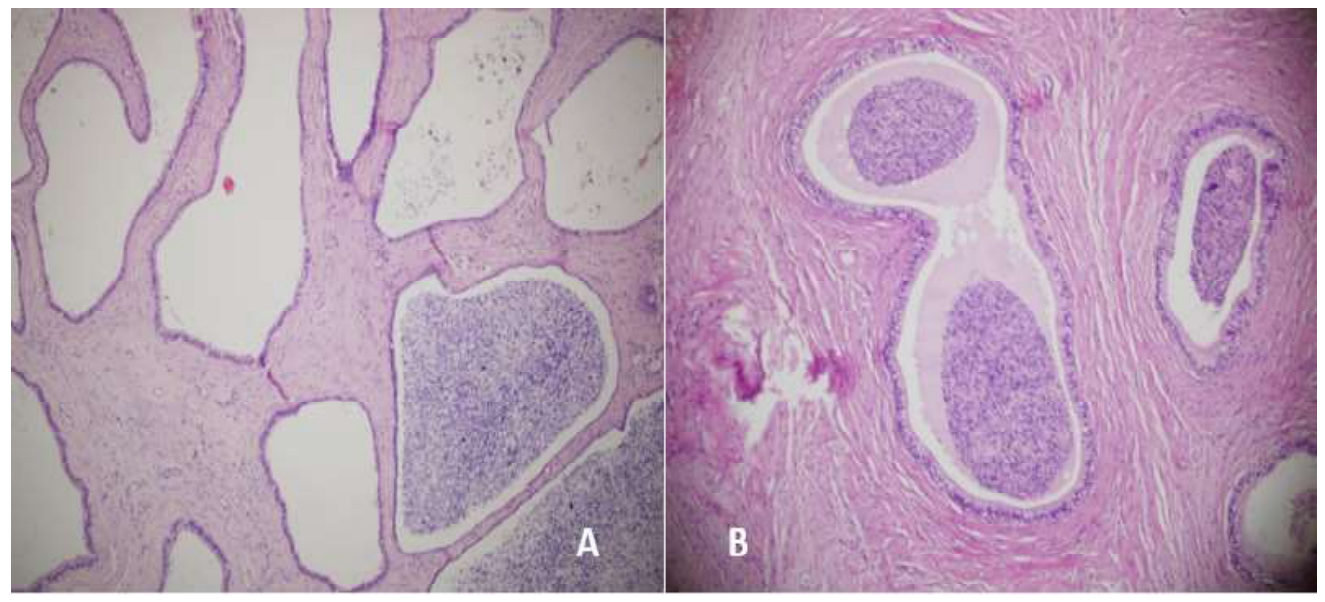

Figura 12: Cola del epidídimo, 56 días después de la aplicación. A: Formaciones quísticas, túnica media y albugínea fibrosada (100x), gluconato de clorhexidina 4\%; B: Fibrosis externa y espermiogranuloma en formación (100x), cloruro de benzalconio al $4 \%+$ cetrimida $0.4 \%$. Tinción Hematoxilina-eosina

El día 56 (Figura 12), ambos grupos (G1 y G2) presentan algunos túbulos con numerosas formaciones quísticas y espermiogranulomas, algunos con fibrosis de la túnica media y de la albugínea; esta última con hemorragia, hemosiderina y fibrosis de moderada a intensa.
Los cambios degenerativos a nivel de la cola del epidídimo que indistintamente suelen presentarse en menor o mayor grado en G1 y G2 desde el día 7 hasta el día 56, corresponden a la pérdida de la arquitectura tubular, formaciones quísticas y presencia de espermiogranulomas; lesiones similares a las repor- 

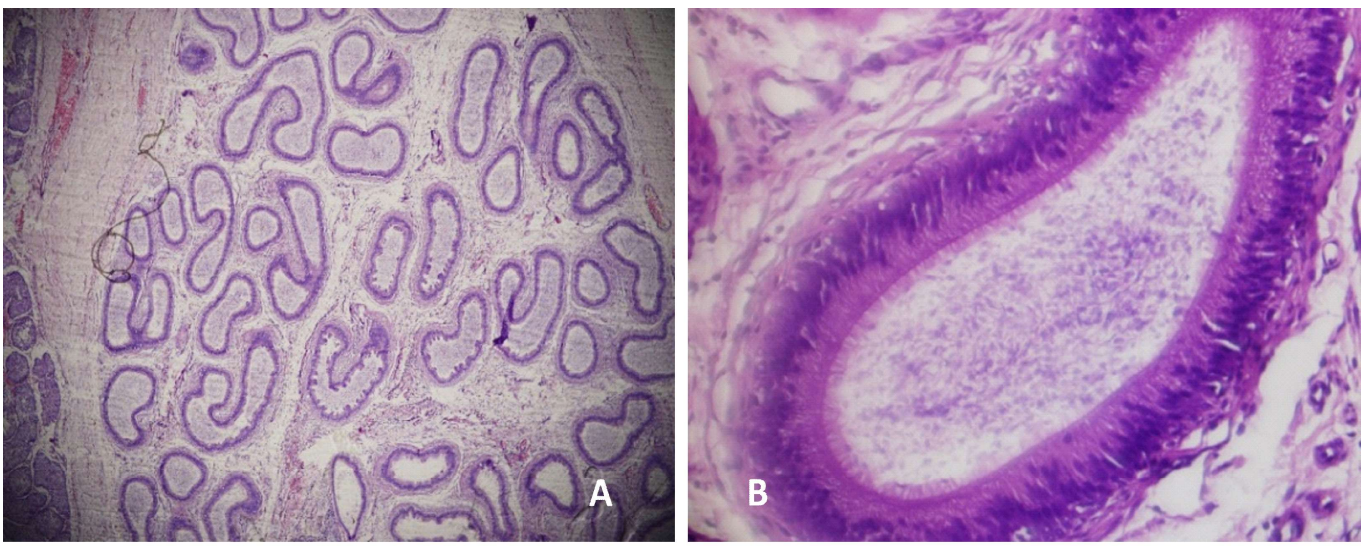

Figura 13. Cola del epidídimo. A: Túbulos epididimarios normales (40x); B: Lumen amplio, estereocilios cortos y espermatozoides dentro del lumen tubular. Tinción Hematoxilinaeosina

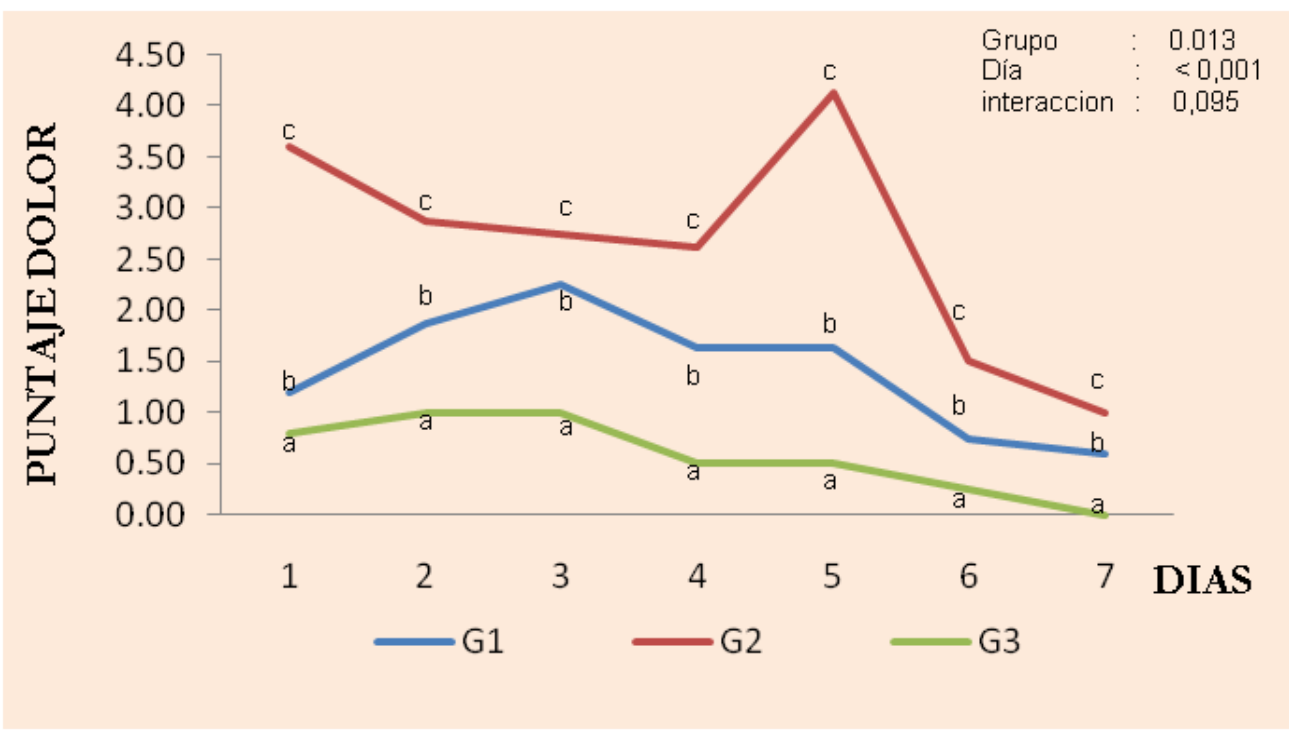

Figura 14. Niveles de dolor experimentados por los perros sometidos a epididimectomía química. G1: gluconato de clorhexidina al 4\%; G2: cloruro de benzalconio al 4\% + cetrimida al $0.4 \%$; G3, Control, cloruro de sodio al $0.9 \%$

tadas en otros estudios (Pineda, 1977; Goicochea et al., 2004), aunque Fahim et al. (1993) no encontró espermiogranulomas en el tejido testicular al realizar la orquiectomía química.

Hacia el final del experimento, las colas epididimarias presentaron una destrucción total de los túbulos e intensa fibrosis (Figura $11 \mathrm{~A}$ ), en forma similar a otros reportes (Pi- neda et al.,1977; Pineda y Hepler, 1978), aunque con la aplicación de gluconato de zinc por vía intratesticular puede ocurrir entre los 60 y 75 días pos-aplicación (Tepsumethanon et al., 2005). Asimismo, la presencia de pigmentos de hemosiderina (Figura 10B) producida de manera secundaria a la hemorragia indica la cronicidad del trastorno. 
En el grupo control no se presentaron lesiones ni a nivel testicular ni a nivel de la cola, cuerpo y cabeza del epidídimo (Figura 13) en todos los cortes histológicos evaluados.

En la medición del grado de algesia se observó un efecto de tratamiento $(\mathrm{p}=0.001)$ y tiempo $(\mathrm{p}<0.001)$, aunque no de interacción $(p=0.9)$ entre los tratamientos y los días de evaluación (Figura 14). El nivel de algesia en los perros sometidos a la epididimectomía química fue diferente a los 7 días de evaluación, presentando un mayor grado de dolor los animales del G1 y G2 (p<0,05) en comparación con el grupo control (G3), siendo el parámetro 'lamido frecuente' el que tuvo el puntaje más alto, debido a una mayor inflamación, necrosis y hemorragia causada por las sustancias químicas aplicadas.

\section{Conclusiones}

- La epididimectomía química mediante la aplicación bilateral e intraepididimaria de gluconato de clorhexidina $4 \%$ o con cloruro de benzalconio al $4 \%+$ cetrimida $0.4 \%$, provocan una intensa fibrosis en la cola del epidídimo con la consecuente azoospermia a partir del día 28, e incremento inicial del volumen testicular e epididimario para retornar a sus volúmenes basales al día 56 .

- La epididimectomía química induce a un mayor nivel de algesia, pero controlable en los perros tratados con benzalconio al $4 \%$ + cetrimida $0.4 \%$, y soportable al gluconato de clorhexidina $4 \%$.

\section{Literatura Citada}

1. Belmar A. 2004. Tramadol y butorfanol asociados acepromazina como método de analgesia preventiva en perras sometidas a ovariohisterectomia. Tesis de Médico Veterinario. Valdivia, Chile: Univ. Austral de Chile. 35 p.
2. Fahim MS, Wang M, Sutcu MF, Fahim Z, Youngquist RS. 1993. Sterilization of dogs with intra-epididymal injection of zinc arginine. Contraception 47: 107-122. doi: 10.1016/0010-7824(93)90113-1

3. Freeman C, Coffey DS. 1993. Sterility in male animals induced by injection of chemical agents into the vas deferens. Fertil Steril 24: 884-890. doi: 10.1016/ S0015-0282(16)40036-1

4. Goicochea VJ, Ureta E, Chavera A. 2004. Control de la capacidad reproduc-tiva del perro mediante la aplicación de sustancias esclerosantes. Inv Valdizana 1: 4-5.

5. Hurber WG 1988. Quimioterapia de las enfermedades microbianas, micóticas y víricas. En: Booth NH, McDonald LC (eds). Farmacología y terapéutica veterinaria. Vol II. Zaragoza, España: Acribia.

6. Immegart HM, Threlfall WR. 2000. Evaluation of intratesticular injection of glycerol for nonsurgical sterilization of dogs. Am J Vet Res 61: 544-549. doi: 10.2460/ajvr.2000.61.544

7. Koger LM. 1978. Calcium chloride castration. Med Vet Pract 59: 119-123.

8. Lambert B. 1951. The frequency of mumps and of mumps orchitis and the consequences for sexuality and fertility. Acta Genet Stat Med 2(Suppl 1): 1-166.

9. Levy JK, Crawford PC, Appel LD, Clifford EL. 2008. Comparison of intratesticular injection of zinc gluconate versus surgical castration to sterilize male dogs. Am J Vet Res 69: 140-143. doi: 10.2460/ajvr.69.1.140

10. Luna I. 1968. Manual of histology methods of the Armed Forces Institute of Pathology. $3^{\text {rd }}$ ed. New York, USA: McGraw-Hills. 249 p.

11. Pineda MH, Hepler DI. 1978. Chemical vasectomy in dogs. Long-term study. Theriogenology 16: 1-11. doi: 10.1016/0093-691x(81)90108-4

12. Pineda MH, Reimers TJ, Faulkner LC, Hopwood ML, Seidel GE. 1977. Azoospermia in dogs induced by injection of sclerosing agents into the caudae of the epididymides. Am J Vet Res 38: 6-12. 
13. Soto FR, Viana WG, Mucciolo GC, Hosomi FY, Vannucchi CI, Mazzei CP, Eyherabide AR, et al. 2009. Evaluation of efficacy and safety of zinc gluconate associated with dimethyl sulphoxide for sexually mature canine males chemical neutering. Reprod Domest Anim 4: 927 931.doi: 10.1111/j.1439-0531.2008.-01119.x
14. Tepsumethanon V, Wilde H, Hemachudha T. 2005. Intratesticular injection of a balanced zinc solution for permanent sterilization of dogs. J Med Assoc Thailand 88: 686-689.

15. Thomson PLM. 2002. Diccionario de especialidades farmacéuticas. $14^{\circ}$ ed. México DF, México, Ediciones PLM. 158p. 